Supporting Information

\title{
Probing lanmodulin's lanthanide recognition via sensitized luminescence yields a platform for quantification of terbium in acid mine drainage
}

Emily R. Featherston, Edward J. Issertell, and Joseph A. Cotruvo, Jr.*

Department of Chemistry, The Pennsylvania State University, University Park, PA 16802 USA

* Corresponding author. E-mail: juc96@psu.edu (J.A.C.).

This file contains Supplementary Methods, Supplementary Tables S1-S4, Supplementary Figures S1-S9, and Supplementary References.

\section{SUPPLEMENTARY TABLES}

Table S1. Primers used in this study

Table S2. Plasmids used in this study

Table S3. Kinetic parameters from the fitted stopped-flow spectrofluorometry data

Table S4. ICP-MS analysis of the acid mine drainage sample

\section{SUPPLEMENTARY FIGURES}

Figure S1. The last residue of EF1 and the first residue of EF2 share a short, common helix Figure S2. Stoichiometric LRET titrations of N87W, T90W, and K94W LanM variants

Figure S3. Stoichiometric LRET titrations of T41W, T65W, and T114W LanM variants Figure S4. Characterization of Trp-LanM upon Eu ${ }^{\text {III }}$ binding

Figure S5. Stoichiometric CD titrations of wild-type and Trp-LanMs with $\mathrm{TbCl}_{3}$

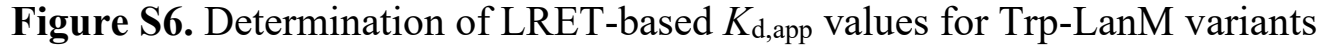

Figure S7. Determination of the luminescence lifetime of $\mathrm{Tb}^{\mathrm{III}}$ in the $\mathrm{T} 90 \mathrm{~W}$ variant

Figure S8. Determination of the luminescence lifetime of $\mathrm{Tb}^{\mathrm{III}}$ in the $\mathrm{K} 94 \mathrm{~W}$ variant

Figure S9. Stopped flow spectrofluorometry of T41W- and T90W-LanM

\section{SUPPLEMENTARY REFERENCES}




\section{SUPPLEMENTARY TABLES}

Table S1. Primers used for cloning of Trp-LanM constructs. ${ }^{a}$

\begin{tabular}{ll}
\hline Name & Sequence \\
\hline N87W-forward & 5'-GGACCCTGACTGGGACGGAACCC-3' \\
N87W-reverse & 5'-AGCTTCTTAAGGTCTGCC-3' \\
T90W-forward & 5'-CAATGACGGATGGCTGGACAAGAAAGAG-3' \\
T90W-reverse & 5'-TCAGGGTCCAGCTTCTTA-3' \\
K94W-forward & 5'-CCTGGACAAGTGGGAGTACTTAGCAGC-3' \\
K94W-reverse & 5'-GTTCCGTCATTGTCAGGG-3' \\
T41W-forward & 5'-CAAAGATGGGTGGATCGATCTGAAAGAGGCTTTGGCGG-3' \\
T41W-reverse & 5'-TCCGGGTCAAACGCCGCG-3' \\
T65W-forward & 5'-TAAAGATGGTTGGCTGGACGCCAAAGAG-3' \\
T65W-reverse & 5'-TCCGGGTCCAACTTGTCG-3' \\
T114W-forward & 5'-CAACGATGGCTGGATTGACGCCCG-3' \\
T114W-reverse & 5'-TCAGGGTTAGCGGCCTTA-3' \\
Sequencing Primers & \\
T7P & 5'-TAATACGACTCACTATAGGG-3' \\
T7T & 5'-GCTAGTTATTGCTCAGCGG-3' \\
\hline
\end{tabular}

${ }^{a}$ Trp mutations are bolded

Table S2. Plasmids used in this study.

\begin{tabular}{lll}
\hline Name & Notes & Source \\
\hline pET-24a & $\mathrm{Km}^{\mathrm{R}}$ & Novagen \\
pET-24a-LanM & $\mathrm{Km}^{\mathrm{R}}$, cytosolic expression & Ref. 1 \\
pET-24a-LanM-T41W & & This work \\
pET-24a-LanM-T65W & & This work \\
pET-24a-LanM-N87W & & This work \\
pET-24a-LanM-T90W & & This work \\
pET-24a-LanM-K94W & & This work \\
pET-24a-LanM-T114W & & This work \\
\hline
\end{tabular}


Table S3. Kinetic parameters from the fitted stopped-flow spectrofluorometry data. Experimental curves were fitted to a single exponential decay $(\mathrm{T} 41 \mathrm{~W})$ and a double exponential decay (T90W, see Figure S9C).

\begin{tabular}{c|cc|cccc}
\hline \multicolumn{9}{c}{ T41W } & \multicolumn{5}{c}{ T90W } \\
\hline $\begin{array}{c}\text { [EGTA }] \\
\mathrm{mM}\end{array}$ & $k_{1}$ & Amplitude & $k_{1}$ & Amplitude & $k_{2}$ & Amplitude \\
\hline 5.00 & $0.0676 \pm 0.0002$ & $2.744 \pm 0.003$ & $0.064 \pm 0.005$ & $2.6 \pm 0.2$ & $0.033 \pm 0.004$ & $1.9 \pm 0.2$ \\
2.50 & $0.0511 \pm 0.0002$ & $2.642 \pm 0.003$ & $0.054 \pm 0.007$ & $2.2 \pm 0.3$ & $0.027 \pm 0.002$ & $2.3 \pm 0.2$ \\
1.25 & $0.0417 \pm 0.0001$ & $2.628 \pm 0.003$ & $0.06 \pm 0.01$ & $1.1 \pm 0.2$ & $0.025 \pm 0.002$ & $2.7 \pm 0.2$ \\
0.63 & $0.0366 \pm 0.0001$ & $2.617 \pm 0.004$ & $0.047 \pm 0.006$ & $1.6 \pm 0.2$ & $0.020 \pm 0.002$ & $2.3 \pm 0.2$ \\
\hline
\end{tabular}


Table S4. ICP-MS analysis of the acid mine drainage sample used for Tb detection, with elements listed in order of atomic number. $\mathrm{V}, \mathrm{Cr}, \mathrm{Zr}$, and $\mathrm{Th}$ were analyzed but not detected.

\begin{tabular}{|c|c|c|}
\hline Element & ppb & Concentration (M) \\
\hline $\mathrm{Li}$ & 145.9 & $2.10 \times 10^{-5}$ \\
\hline $\mathrm{Be}$ & 11.98 & $1.33 \times 10^{-6}$ \\
\hline $\mathrm{Na}$ & 3994 & $1.74 \times 10^{-4}$ \\
\hline $\mathrm{Mg}$ & 233000 & $9.59 \times 10^{-3}$ \\
\hline $\mathrm{Al}$ & 18940 & $7.02 \times 10^{-4}$ \\
\hline $\mathrm{Si}$ & 11530 & $4.10 \times 10^{-4}$ \\
\hline $\mathrm{K}$ & 3217 & $8.23 \times 10^{-5}$ \\
\hline $\mathrm{Ca}$ & 3109 & $7.76 \times 10^{-5}$ \\
\hline Sc & 2.85 & $6.33 \times 10^{-8}$ \\
\hline $\mathrm{Ti}$ & 292.2 & $6.10 \times 10^{-6}$ \\
\hline $\mathrm{Mn}$ & 24790 & $4.51 \times 10^{-4}$ \\
\hline $\mathrm{Fe}$ & 424.2 & $7.60 \times 10^{-6}$ \\
\hline $\mathrm{Co}$ & 481.1 & $8.16 \times 10^{-6}$ \\
\hline $\mathrm{Ni}$ & 736.6 & $1.25 \times 10^{-5}$ \\
\hline $\mathrm{Cu}$ & 18.23 & $2.87 \times 10^{-7}$ \\
\hline $\mathrm{Zn}$ & 1454 & $2.22 \times 10^{-5}$ \\
\hline $\mathrm{Sr}$ & 53.01 & $6.05 \times 10^{-7}$ \\
\hline $\mathrm{Y}$ & 93.35 & $1.05 \times 10^{-6}$ \\
\hline $\mathrm{Cd}$ & 1.27 & $1.13 \times 10^{-8}$ \\
\hline $\mathrm{Ba}$ & 8.61 & $6.27 \times 10^{-8}$ \\
\hline $\mathrm{La}$ & 14.18 & $1.02 \times 10^{-7}$ \\
\hline $\mathrm{Ce}$ & 45.84 & $3.27 \times 10^{-7}$ \\
\hline $\operatorname{Pr}$ & 7.10 & $5.04 \times 10^{-8}$ \\
\hline $\mathrm{Nd}$ & 36.44 & $2.53 \times 10^{-7}$ \\
\hline $\mathrm{Sm}$ & 12.40 & $8.25 \times 10^{-8}$ \\
\hline $\mathrm{Eu}$ & 3.56 & $2.35 \times 10^{-8}$ \\
\hline $\mathrm{Gd}$ & 20.71 & $1.32 \times 10^{-7}$ \\
\hline $\mathrm{Tb}$ & 3.32 & $2.09 \times 10^{-8}$ \\
\hline Dy & 18.87 & $1.16 \times 10^{-7}$ \\
\hline Ho & 3.53 & $2.14 \times 10^{-8}$ \\
\hline $\mathrm{Er}$ & 9.31 & $5.56 \times 10^{-8}$ \\
\hline $\mathrm{Tm}$ & 1.08 & $6.42 \times 10^{-9}$ \\
\hline $\mathrm{Yb}$ & 6.68 & $3.86 \times 10^{-8}$ \\
\hline $\mathrm{Lu}$ & 0.88 & $5.01 \times 10^{-9}$ \\
\hline $\mathrm{Pb}$ & 1.37 & $6.63 \times 10^{-9}$ \\
\hline $\mathrm{U}$ & 1.48 & $6.22 \times 10^{-9}$ \\
\hline
\end{tabular}




\section{SUPPLEMENTARY FIGURES}

Figure S1. The last residue of EF1 (Glu46) and the first residue of EF2 (Asp59) share a short, common helix. Metal coordinating residues are shown as sticks, and the EF hands are shown in gray. Alpha helices of this length are often poised between order and disorder, ${ }^{2}$ suggesting the possibility that metal binding to one EF hand and concomitant loop stabilization could be communicated to the other EF hand.

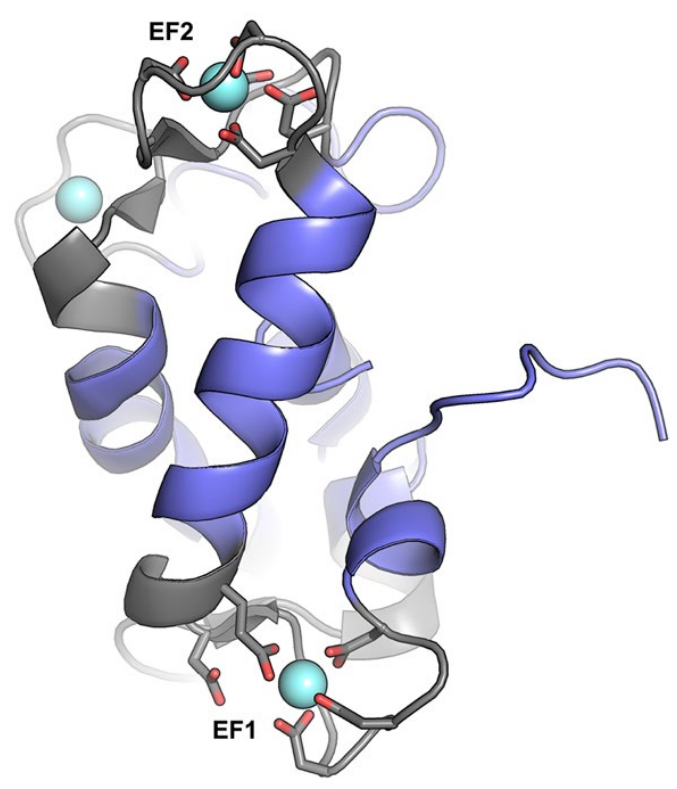


Figure S2. Preliminary stoichiometric LRET titrations of LanM proteins containing Trp substitutions at (A) N87W (B) T90W and (C) K94W. (D-F) Expanded 515-575 nm range to highlight the emission feature at $\sim 545 \mathrm{~nm}$ for each construct. Note that N87W and K94W exhibit increases in Trp fluorescence upon metal binding but minimal $\mathrm{Tb}^{\mathrm{III}}$ emission, whereas $\mathrm{T} 90 \mathrm{~W}$ exhibits quenching of Trp emission along with efficient energy transfer to $\mathrm{Tb}^{\mathrm{III}}$. Experimental parameters: $280 \mathrm{~nm}$ excitation, $400-700 \mathrm{~nm}$ emission, $5 \mathrm{~nm}$ excitation and emission slit widths, $120 \mathrm{~nm} / \mathrm{min}$ scan rate, $1 \mathrm{~nm}$ data interval, 250-395 $\mathrm{nm}$ excitation filter, 430-1100 $\mathrm{nm}$ emission filter.

A

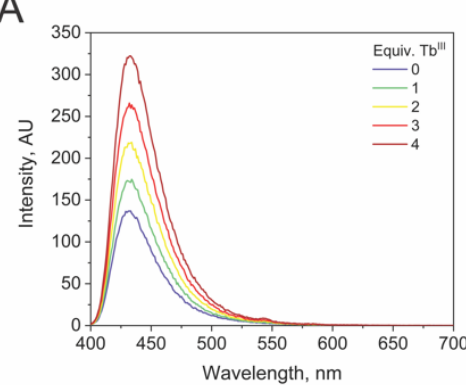

D

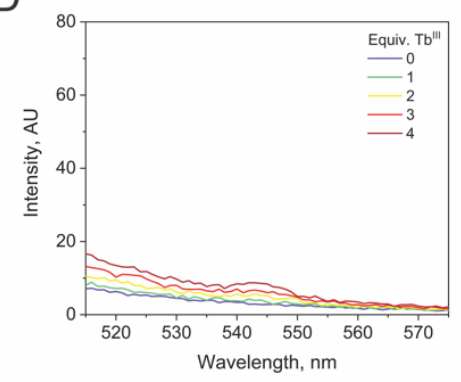

B

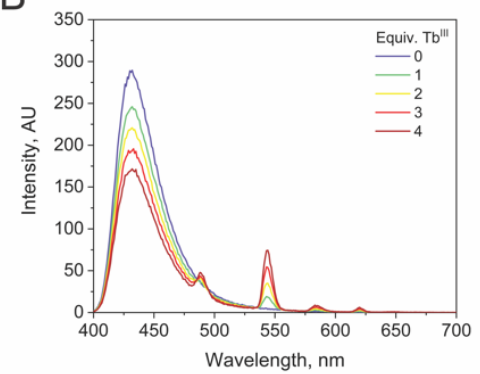

$E$

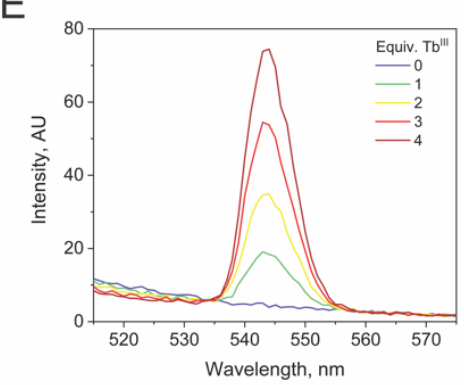

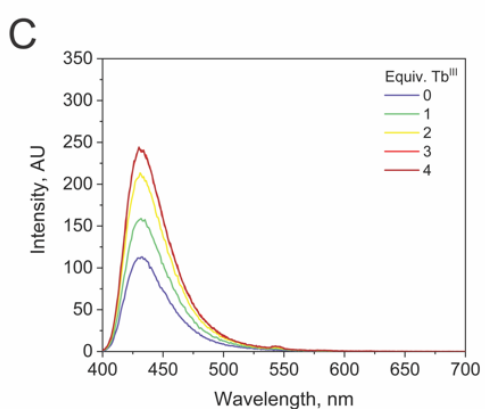

F

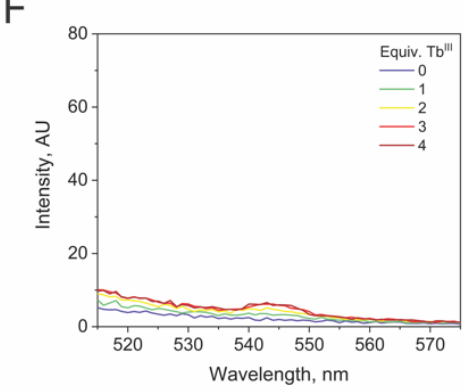


Figure S3. Stoichiometric LRET titrations of LanM proteins containing Trp substitutions at (A) T41W (B) T65W and (C) T114W. (D-F) Expanded 515-575 $\mathrm{nm}$ range to highlight the emission feature at $\sim 545 \mathrm{~nm}$ for each construct. Experimental parameters: $280 \mathrm{~nm}$ excitation, $400-700 \mathrm{~nm}$ emission, $5 \mathrm{~nm}$ excitation and emission slit widths, $120 \mathrm{~nm} / \mathrm{min}$ scan rate, $1 \mathrm{~nm}$ data interval, 250$395 \mathrm{~nm}$ excitation filter, 430-1100 nm emission filter.
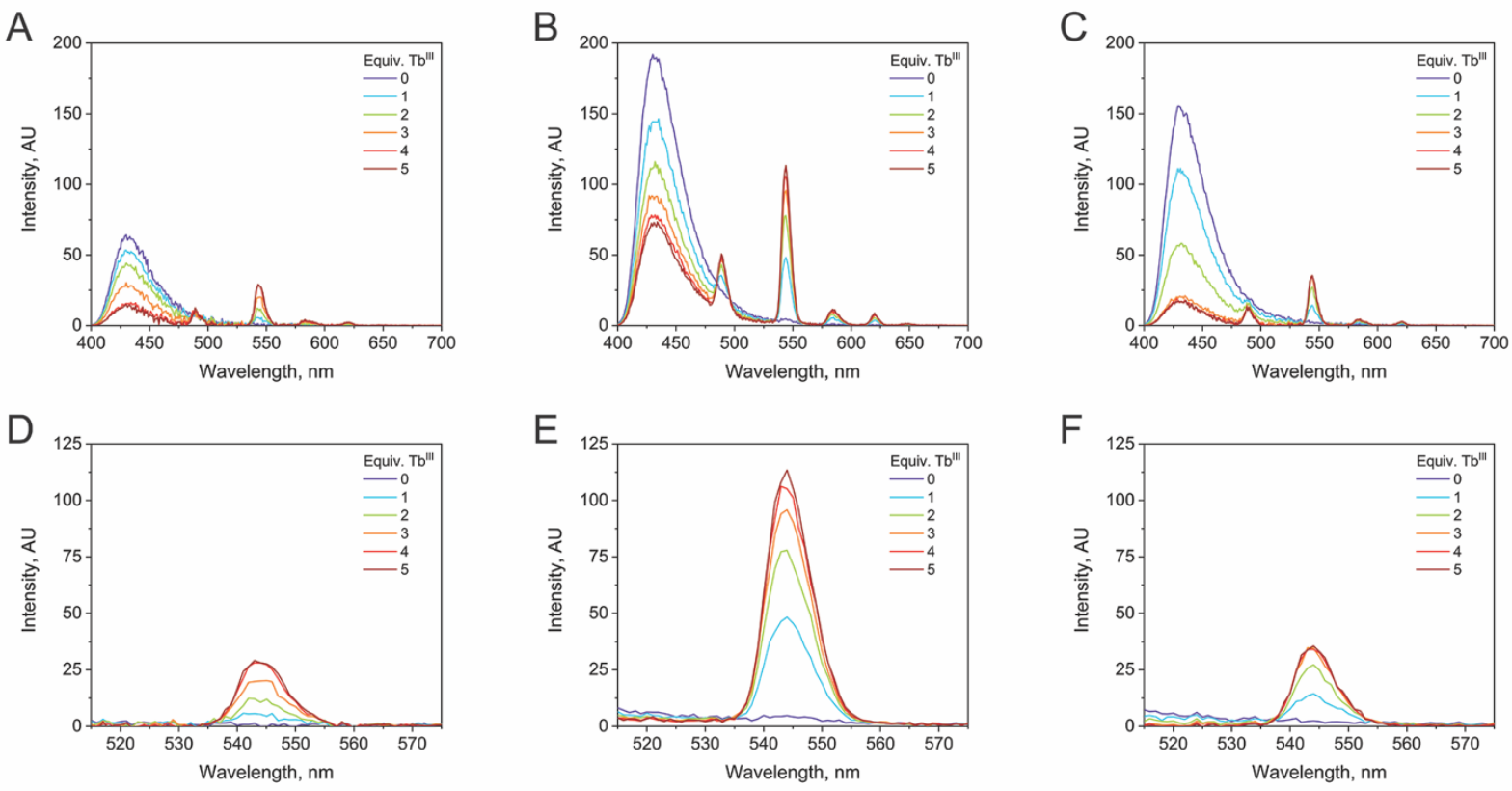
Figure S4. Characterization of Trp-LanM (T41W) upon $\mathrm{Eu}^{\mathrm{III}}$ binding monitored by spectrofluorometry. Quenching of the Trp emission was observed in the presence of Eu ${ }^{\text {III }}$, but significant sensitized $\mathrm{Eu}^{\mathrm{III}}$ emission features were not observed. The failure to observe significant Trp-sensitized $\mathrm{Eu}^{\mathrm{III}}$ emission under these conditions is consistent with other protein systems. ${ }^{3}$ Experimental parameters: $275 \mathrm{~nm}$ excitation, 400-700 nm emission, 250-395 $\mathrm{nm}$ excitation filter, 430-1100 nm emission filter.

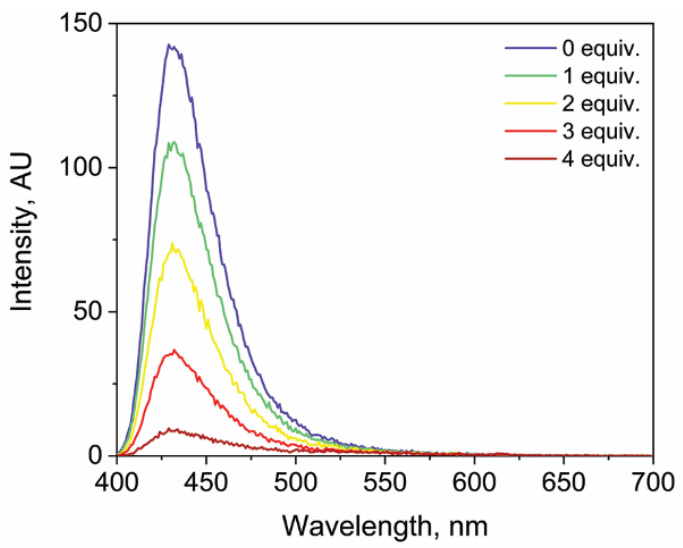


Figure S5. Stoichiometric titrations of $15 \mu \mathrm{M}$ wild-type, T41W, T65W, N87W, T90W, K94W, and $\mathrm{T} 114 \mathrm{~W}$ LanMs with $\mathrm{TbCl}_{3}$, followed using $\mathrm{CD}$ spectroscopy. The disruption of the conformational response of $\mathrm{T} 65 \mathrm{~W}$ is particularly noteworthy.
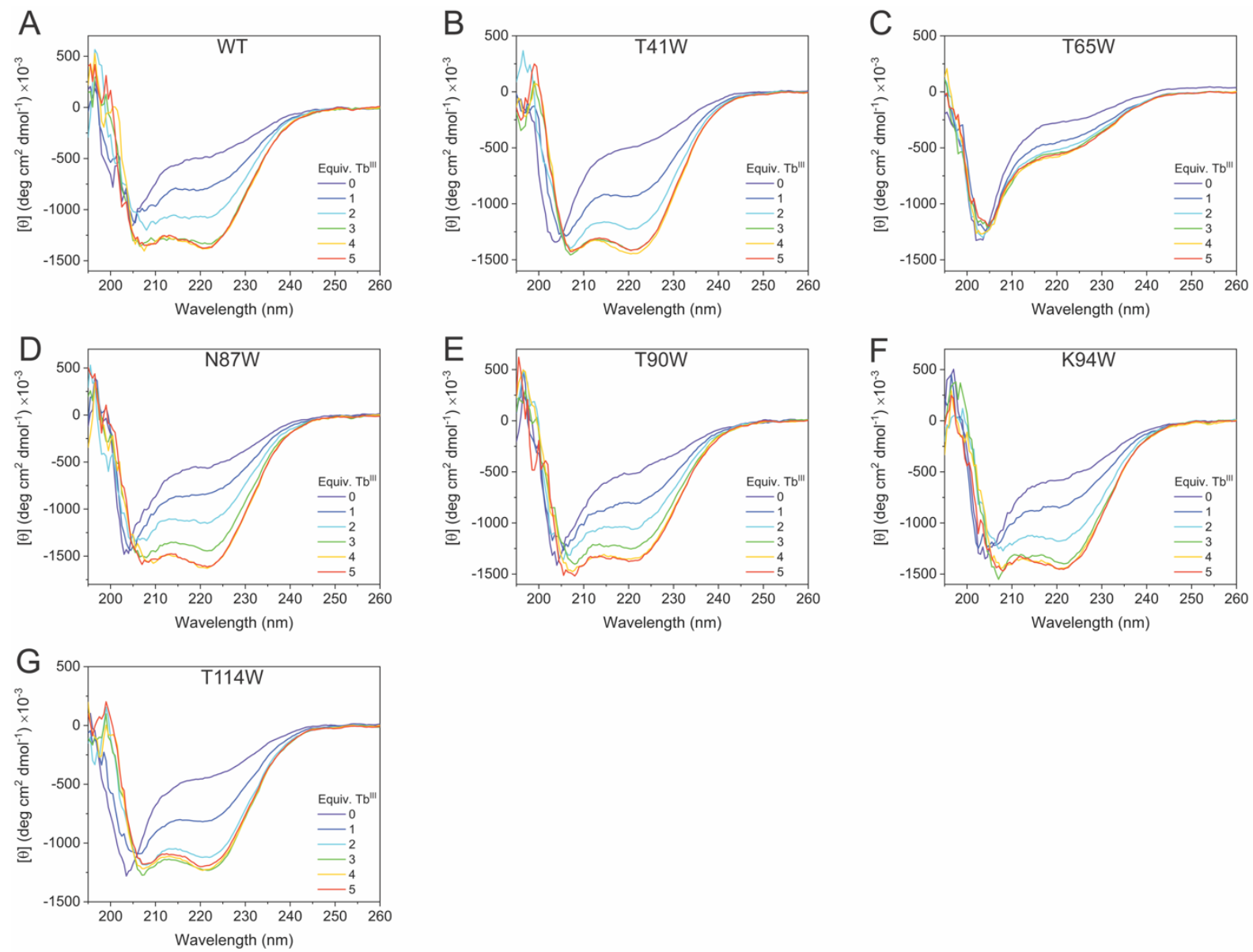
Figure S6. Representative titration curves from determination of LRET-based $K_{\text {d,app }}$ values for 10 $\mu \mathrm{M}$ (A) T41W (B) T90W (C) K84W (D) T114W LanM variants. Tb ${ }^{\mathrm{III}}$ emission was monitored from 400-700 nm with at a fixed excitation at $295 \mathrm{~nm}$, at various EDDS-buffered free $\mathrm{Tb}^{\mathrm{III}}$ ion concentrations. Luminescence intensities at $544-546 \mathrm{~nm}$ were averaged and, to remove the contribution from Tb-EDDS luminescence, values from a control experiment in the absence of protein were subtracted. The resulting values were fitted to the Hill equation to determine $K_{\mathrm{d} \text {,app }}$ and $n$, which are given in Table 1.

A

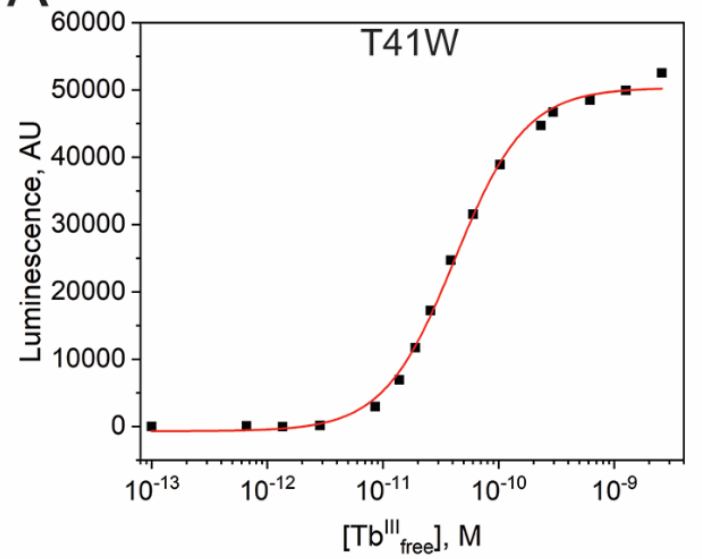

C

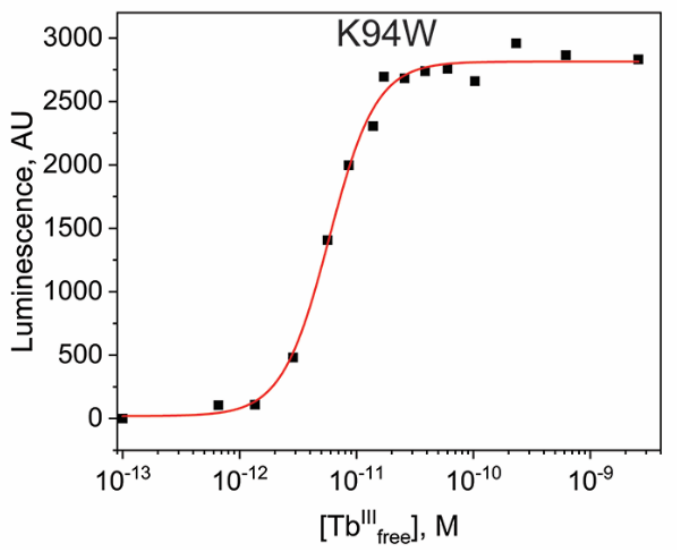

$\mathrm{B}$

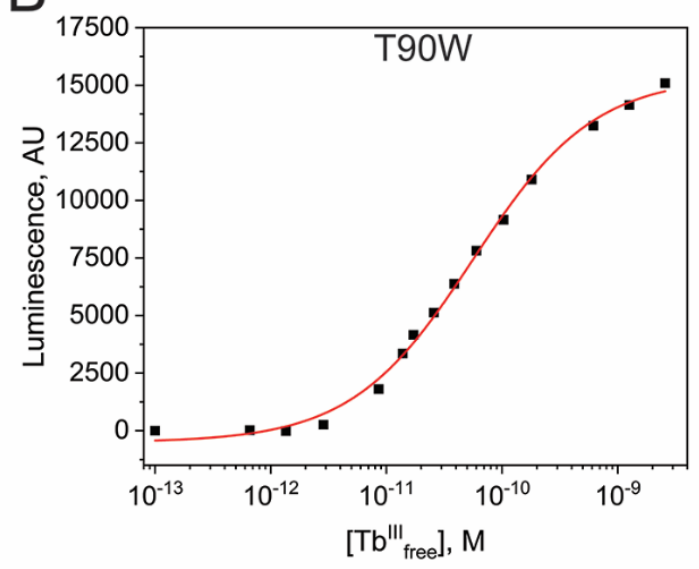

D

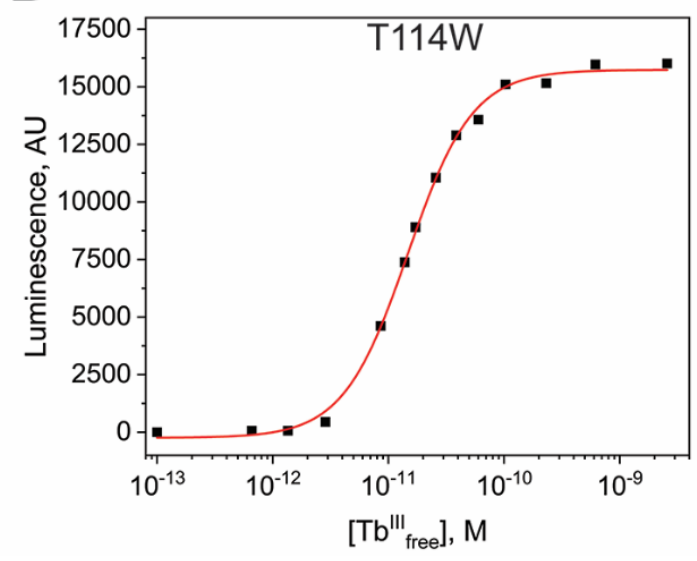


Figure S7. Determination of the luminescence lifetime of $\mathrm{Tb}^{\mathrm{III}}$ in the T90W variant. A) T90WLanM luminescence decay curves in $0,25,50$, and $75 \% \mathrm{D}_{2} \mathrm{O}$. The data from each curve (carried out in triplicate) were fitted to a single exponential decay. B) Linear dependence of the inverse decay time constant at different mole fractions of $\mathrm{D}_{2} \mathrm{O} .1 / \tau\left(\mathrm{D}_{2} \mathrm{O}\right)$ was determined from the $\mathrm{y}$ intercept.
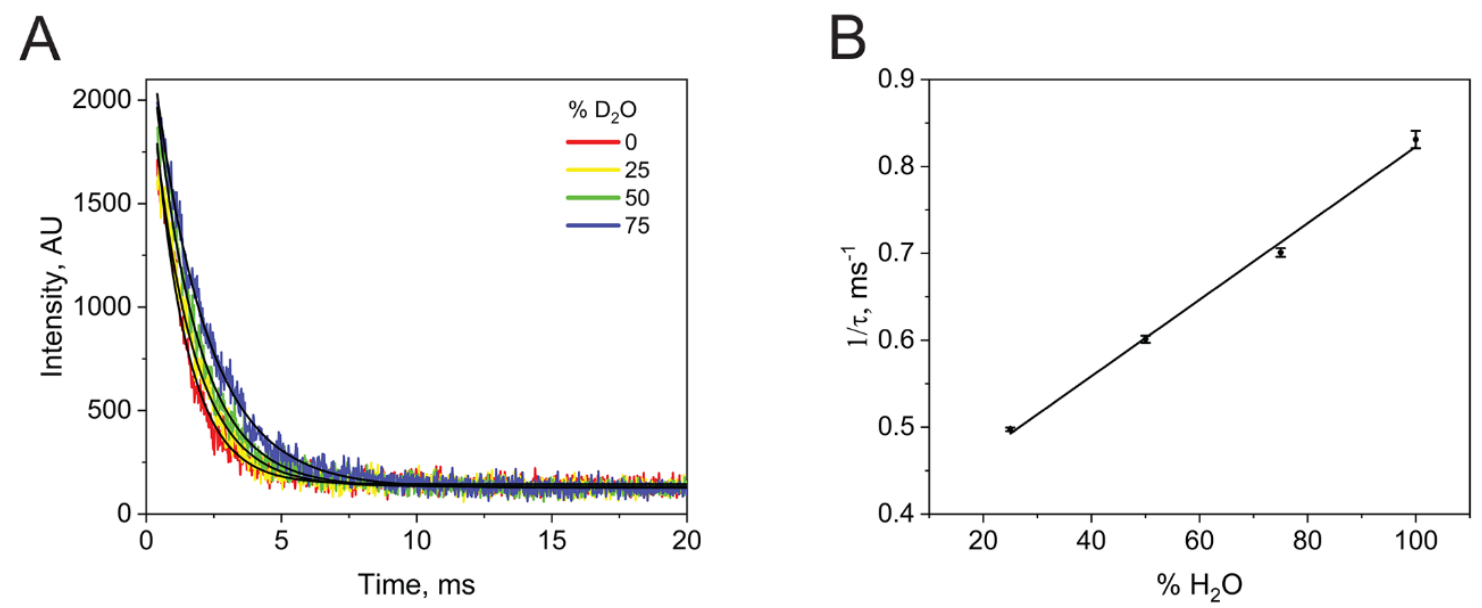
Figure S8. Determination of the luminescence lifetime of $\mathrm{Tb}^{\mathrm{III}}$ in the $\mathrm{K} 94 \mathrm{~W}$ variant. A) K94WLanM luminescence decay curves in $0,25,50$, and $75 \% \mathrm{D}_{2} \mathrm{O}$. The data from each curve (carried out in triplicate) were fitted to a single exponential decay. Because of the low luminescence intensity of this variant, these experiments were carried out at $30 \mu \mathrm{M}$ protein. B) Linear dependence of the inverse decay time constant at different mole fractions of $\mathrm{D}_{2} \mathrm{O}, 1 / \tau\left(\mathrm{D}_{2} \mathrm{O}\right)$ was determined from the y-intercept.
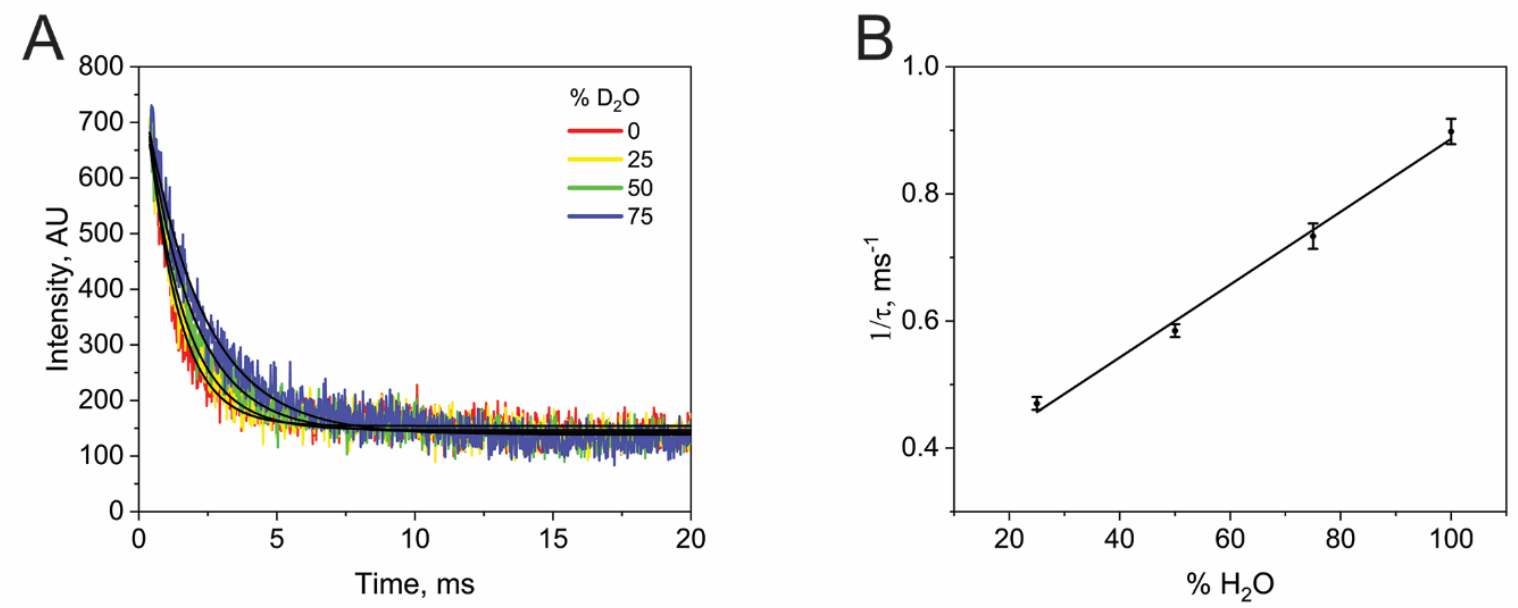
Figure S9. Stopped flow spectrofluorometry of T41W- and T90W-LanM. A) T41W stopped-flow traces including single exponential fits (black lines). B) T90W stopped-flow traces including double exponential fits (black lines). C) Representative residual from the single exponential fitting of a trace from the T90W $5 \mathrm{mM}$ EGTA data set. D) Representative residual from the double exponential fitting of the same trace, showing the necessity of the double exponential fit. There was not a full decrease in fluorescence over the measured time periods, likely due to some Trp fluorescence reaching the detector even with the $450 \mathrm{~nm}$ LP filter.
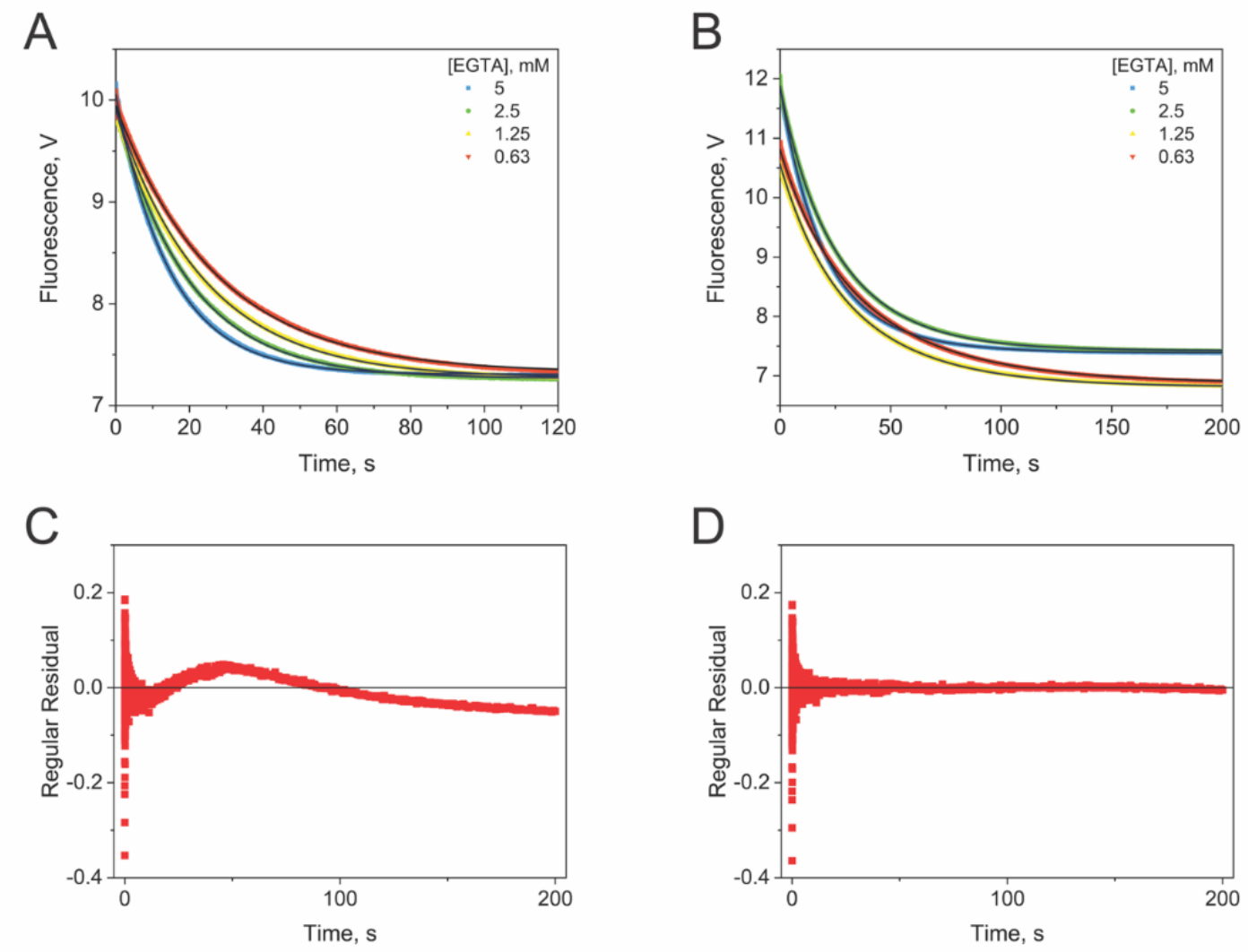

\section{SUPPLEMENTARY REFERENCES}

1. Featherston, E. R.; Mattocks, J. A.; Tirsch, J. L.; Cotruvo, J. A., Jr., Heterologous expression, purification, and characterization of proteins in the lanthanome. Methods Enzymol. 2021, 650, 119-157.

2. Sandhu, K. S.; Dash, D., Dynamic $\alpha$-helices: Conformations that do not conform. Proteins 2007, 68, 109-122.

3. Horrocks, W. D.; Sudnick, D. R., Lanthanide ion luminescence probes of the structure of biological macromolecules. Acc. Chem. Res. 1981, 14, 384-392. 